\title{
Methylphenidate and Cognitive Flexibility: Dissociated Dose Effects in Hyperactive Children
}

\author{
Rosemary Tannock, ${ }^{1,3}$ Russell Schachar, ${ }^{1}$ and Gordon Logan ${ }^{2}$
}

A randomized, double-blind, placebo-controlled trial was conducted to assess the acute effects of placebo and three doses of methylphenidate (MPH) (0.3, $0.6,0.9 \mathrm{mg} / \mathrm{kg}$ ) on cognitive flexibility and overt behavior in 28 children with a confirmed diagnosis of attention deficit-hyperactivity disorder. Two underlying cognitive processes (response inhibition and response reengagement) were assessed by measuring the probability and speed with which subjects could inhibit responses to a primary task (forced-choice letter discrimination) and immediately execute a response to a secondary task (simple reaction time) when given a signal to do so. Results indicated that MPH enhanced cognitive flexibility, although the high dose was less effective than lower doses in enhancing response inhibition. Dissociations of dose effects on cognitive function and behavior were demonstrated: Dose-response functions for changes in behavior were linear, whereas the function for response inhibition was U-shaped. Findings argue against the typical clinical practice of determining the response to stimulant treatment from a single measure such as parent report of child behavior.

Stimulant medication, such as methylphenidate (MPH), is the most widely used treatment for children with attention deficit-hyperactivity disorder (ADHD) (Safer \& Krager, 1988). The primary objective of this treatment is to reduce the core behavioral symptoms of ADHD (inattention, impul-

Manuscript received in final form May 2, 1994.

This research was supported by funds from the Medical Research Council of Canada and Health and Welfare Canada. The authors would like to express their appreciation to Dr. Abel Ickowicz for conducting the diagnostic assessments and feedback sessions, and to Patricia Fulford and Karen Purvis for their valuable assistance. Also, we thank the anonymous reviewers for their helpful comments on an earlier version of the manuscript.

${ }^{1}$ Department of Psychiatry, The Hospital for Sick Children, Toronto, Canada.

${ }^{2}$ Department of Psychology, University of Illinois, Champaign, Illinois.

${ }^{3}$ Address all correspondence to Rosemary Tannock, Ph.D., Psychiatry Research Unit, Department of Psychiatry, The Hospital for Sick Children, 555 University Avenue, Toronto, Ontario, Canada, M5G 1X8. 
siveness, and overactivity). The beneficial effects of stimulants on these overt behaviors are well documented and improvements are dose-dependent, with higher doses typically producing greater changes than lower doses (see DuPaul \& Barkley, 1990; Jacobvitz, Sroufe, Stewart, \& Leffert, 1990; Rapport \& Kelly, 1991; Schachar \& Tannock, 1993, for a review). By contrast, stimulant effects on cognitive function are less clear (Rapport \& Kelly, 1991). One issue of concern is whether stimulants have concomitant effects on cognitive function that are deleterious and may impair rather than improve learning (American Academy of Pediatrics, 1987). It is not known which aspects of cognitive function may be at risk, but one hypothesis is that stimulants impair cognitive flexibility and produce perseveration of thought and action (Peters, Dykman, Ackerman, \& Romine, 1974; Robbins \& Sahakian, 1979; Solanto, 1984; Sroufe \& Stewart, 1973; Swanson \& Kinsbourne, 1979). Cognitive flexibility refers to the ability to shift freely from one concept to another or change a course of action or thought according to the demands of a new situation (Lezak, 1983; Logan \& Cowan, 1984; Walsh, 1978).

A wide range of phenomena resulting from stimulant treatment have been described that are consistent with the cognitive flexibility hypothesis: a decreased ability to shift mental set (Dyme, Sahakian, Golinko, \& Rabe, 1982; Tannock \& Schachar, 1992); repetitive scanning of restricted areas of a visual display without improvement in performance on a match-tosample task (Flintoff, Barron, Swanson, Ledlow, \& Kinsbourne, 1982); problems thinking divergently as opposed to convergently (Solanto \& Wender, 1989); motor stereotypy (Robbins \& Sahakian, 1979); and clinical descriptions of children treated with stimulants as looking like "zombies" (Sprague \& Gadow, 1976), "unusually inactive, not simply less restless" (Rapoport et al., 1978, p. 562), or fixated to whatever they were doing (Laufer, Denhoff \& Riverside, 1957).

On the other hand, the extant evidence of negative effects of stimulants on cognitive flexibility is weak and inconsistent. For example, the aforementioned putative negative effects have been associated primarily with high doses of stimulants (i.e., doses approximating $1.0 \mathrm{mg} / \mathrm{kg}$ ), and have been transient (Tannock \& Schachar, 1992), demonstrated in very small samples or in a subset of a larger sample (Dyme et al., 1982; Solanto \& Wender, 1989), and not confirmed by statistical analyses (Brown \& Sleator, 1979; Solanto \& Wender, 1989; Sprague \& Sleator, 1977; Tannock \& Schachar, 1992). Also, it is important to note that many investigators have found the effects of stimulants to be beneficial for performance on a range of cognitive and academic tasks that require cognitive flexibility (Douglas, Barr, Amin, O'Neill, \& Britton, 1988; Pelham, Bender, Caddell, Booth, \& Moorer, 1985; Rapport et al., 1988; Solanto \& Connors, 1982; Tannock, 
Schachar, Carr \& Logan, 1989b). Nevertheless, in view of the large numbers of children potentially at risk from any negative effects of stimulants and the inconsistency of research findings, further investigation of the cognitive flexibility hypothesis is warranted.

If adverse effects of stimulants on cognitive flexibility can be confirmed, it will become important to identify and characterize the processes that underlie the phenomena and to understand how they are regulated. A fundamental problem with most of the previous studies is that the methods employed did not isolate and measure the various cognitive processes underlying performance. Thus, it is not clear which processes may be adversely affected by stimulant medication.

Research with adults and normal children indicates that "reactive flexibility," which refers to the ability to switch rapidly and appropriately from one thought or action to another (Grattan \& Eslinger, 1990), involves two sets of processes: namely, the ability to inhibit an ongoing action or response (response inhibition) and the ability to execute an alternative response following inhibition of the current action (response reengagement) (Logan \& Burkell, 1986; Logan \& Cowan, 1984; Logan, Cowan, \& Davis, 1984). Thus, the observed perseverative phenomenology associated with stimulant treatment may result from impairments in one or both of these sets of processes.

In a previous dose-response study (Tannock, Schachar, Carr, Chajczyk, \& Logan, 1989a), we used a stop signal paradigm that is a well-established and theoretically derived method for studying the central regulation of the ability to inhibit a planned action or thought (Logan \& Cowan, 1984; Logan et al., 1984; Schachar \& Logan, 1990). The "checked swing" phenomenon in baseball or cricket provides a useful analogy for this inhibitory action (De Jong, Coles, Logan, \& Gratton, 1990; Osman, Kornblum, \& Meyer, 1990). Of relevance here are the findings that children with ADHD exhibit deficits in response inhibition (Pliszka \& Borcherding, 1994; Schachar \& Logan, 1990; Schachar, Tannock, \& Logan, 1993; Schachar, Tannock, Marriott, \& Logan, in press), so that any further impairment in this cognitive process as a result of stimulant treatment would be of great concern. We found that stimulants enhanced the response inhibition processes (Tannock et al., 1989a). Moreover, since improvements were greater at high dose $(1.0 \mathrm{mg} / \mathrm{kg})$ than at low dose $(0.3 \mathrm{mg} / \mathrm{kg})$, this beneficial effect appeared to be dose-dependent. However, the absence of a moderate dose prevented us from concluding that dose effects on inhibition were linear. More importantly, that method did not provide a measure of the second component of cognitive flexibility - the ability to rapidly execute an alternative response after inhibiting the ongoing response (i.e., response reengagement). 
The purpose of the present study was to use a modification of the stop signal paradigm - the change paradigm (Logan \& Burkell, 1986) - in order to assess dose (low medium, high) effects of MPH on the two sets of cognitive processes (response inhibition and response reengagement) that underlie cognitive flexibility. If stimulant medication has a deleterious effect on cognitive flexibility, it could do so by impairing either the inhibitory processes or the response reengagement processes, or both mechanisms. Although findings from our previous study militate against adverse effects on the inhibitory processes, it is possible that the increased cognitive demand of the change paradigm (i.e., the additional demand of producing an overt response to the stop signal) might alter the dose-response relationship. Thus, we argued that adverse effects of stimulant medication on cognitive flexibility would be evident if either one or both sets of processes (response inhibition, response reengagement) were impaired by at least one dose of MPH relative to placebo.

A second objective of the study was to investigate the hypothesized dissociation between dose effects on behavior and cognitive function that was proposed by Sprague and Sleator (1977). Noting a disparity between the stimulant dose that maximally improved behavior and that which optimized performance on a short-term memory task, Sprague and Sleator concluded that behavioral and cognitive changes have different dose-response relationships: The dose-response curve for behavior (and for cardiovascular function) was thought to be linear, whereas that for cognition was U-shaped (i.e., nonlinear), indicating an optimal dosage above which performance would deteriorate with further increases in dose. In the present study, we measured dose effects on behavior at the same time that we measured the effects on cognitive function. Evidence of differences in the shape of dose-response curves for different measures of behavior and cognitive function would argue against the typical clinical practice of determining the response to stimulant treatment from a single measure, such as parent or teacher report of child behavior.

\section{METHODS}

\section{Subjects}

Twenty-eight children ( 25 boys, 3 girls) with ADHD participated in the study with signed written statements of informed consent from parents and verbal assent from children. They were all of average or above average intelligence (mean full-scale Wechsler Intelligence Scale for Children-Revised (WISC-R; Wechsler, 1976) score $=106.5, S D=15.6$ ), with a mean age of 
8.9 years $(S D=1.2)$. Children were recruited for the project from children referred to the outpatient psychiatry department in a children's hospital serving a large metropolitan area, for evaluation of their responses to stimulant treatment. Children were excluded from this study if they did not meet DSMIII-R diagnostic criteria for ADHD (American Psychiatric Association, 1987), had a WISC-R score of less than 80 , met DSM-III-R criteria for an anxiety disorder, or exhibited a major neurological, physical, or sensory impairment.

Diagnosis was based upon the results of semistructured diagnostic interviews with the child's parent and teacher (Parent Interview for Child Symptoms - Revised, Schachar \& Wachsmuth, 1989; Teacher Telephone Interview, Schachar \& Tannock, 1990). In both interviews, the clinician elicits from the informant descriptions of the child's behavior in various situations, specified by the interview (e.g., playing outdoors, playing indoors with a friend, watching television). The clinician, rather than the informant, rates the behavior on a 4-point scale ( 0 to 3 ) by referring to specific criteria about the severity, frequency, age-appropriateness, and level of disability resulting from the behavior (only behavior rated as 2 or 3 is counted as a symptom). Interrater reliability and convergent validity of these interviews have been reported previously and have been high (Schachar et al., in press). Parents and teachers also completed the appropriate versions of the Rutter Child Scales (Rutter, Tizard, \& Whitmore, 1970/1981), the Conners Abbreviated Symptom Questionnaire (Conners, 1973) and the Swanson, Nolan, and Pelham (SNAP) Checklist for ADDH symptoms based on DSM-III criteria (Pelham, Atkins, and Murphy, 1981). Diagnostic characteristics of the sample are shown in Table I.

The majority of children in the sample (23 of the 28 ) were considered to have pervasive $\mathrm{ADHD}$, because they met diagnostic criteria both at home and school (i.e., on both parent and teacher interviews). The remaining children met diagnostic criteria on one interview but met less than five criteria on the other. Ten children in the sample (35\%) also met diagnostic criteria for conduct disorder or moderate to severe oppositional disorder, and $11(39 \%)$ exhibited learning disabilities. ${ }^{4}$ Six children (20\%) had been receiving stimulant medication prior to the present study, but their regular doses were discontinued at least 48 hours before the diagnostic assessment and were not reinstated until the 5 -day trial had been completed. The children came predominantly from middle- and upper-middle-class families, although the precise socioeconomic status of the families was not recorded upon entry into the study. The majority of the children were Caucasian

${ }^{4}$ Learning disability was defined by a standardized score on either the reading or arithmetic subtests (or both) of the Wide Range Achievement Test - Revised (Jastak \& Wilkinson, 1984) that was at least $1.5 S D$ below the mean for age and at least $1 S D$ below the full-scale IQ score on the Wechsler Intelligence Scale for Children-Revised. 
Table I. Diagnostic Characteristics of the Sample $(\mathrm{N}=28)$

\begin{tabular}{|c|c|c|}
\hline & Parent & Teacher \\
\hline & Mean $(S D)$ & Mean $(S D)$ \\
\hline \multicolumn{3}{|l|}{$\overline{\text { Diagnostic interview }}{ }^{a}$} \\
\hline ADHD symptoms & $9.8(2.6)$ & $9.1(2.6)$ \\
\hline ODD symptoms & $4.8(2.8)$ & $3.2(2.8)$ \\
\hline CD symptoms & $0.8(1.0)$ & $1.6(2.2)$ \\
\hline \multicolumn{3}{|l|}{ Rutter Child Scales $^{b}$} \\
\hline Total score & $21.1(7.3)$ & $16.7(7.8)$ \\
\hline Emotional Factor & $2.9(1.9)$ & $2.4(2.1)$ \\
\hline Aggressive/Antisocial Factor & $4.9(2.8)$ & $4.2(3.6)$ \\
\hline Hyperactive Factor & $4.4(1.5)$ & $4.6(1.8)$ \\
\hline Conners Abbreviated Symptom Questionnaire ${ }^{c}$ & $21.3(5.8)$ & $17.2(5.7)$ \\
\hline \multicolumn{3}{|l|}{ SNAP Checklist ${ }^{d}$} \\
\hline Impulsive symptoms & $4.4(1.5)$ & $3.8(2.0)$ \\
\hline Inattentive symptoms & $4.6(0.5)$ & $3.5(1.7)$ \\
\hline Hyperactive symptoms & $3.8(1.9)$ & $3.1(1.9)$ \\
\hline
\end{tabular}

${ }^{a}$ Mean number of DSM-III-R symptoms of attention deficit-hyperactivity disorder (ADHD), oppositional deficit disorder (ODD), and conduct disorder (CD) endorsed by the clinician based in the Parent Interview for Child Symptoms - Revised (Schachar \& Wachsmuth, 1989) and the Teacher Telephone Interview (Schachar \& Tannock, 1990).

${ }^{b} A$ total score of 13 or more on the parent scales and 9 on the teacher scales is predictive of clinical diagnosis of a psychiatric disorder (Rutter et al., 1970/1981); factor scores of 5 or more, which are obtained by $3 \%$ of 10 -year-old boys are considered clinically important (Schachar, Rutter, \& Smith, 1981).

'A score of 16, which is equivalent to a $T$ score of 70 (i.e., $2 S D$ above the mean for 8- to 9 -year old boys) is considered indicative of psychopathology.

${ }^{d}$ Swanson, Nolan, and Pelham (SNAP) Checklist; Number of DSM-III symptoms rated as pretty much or very much.

$(90 \%)$ and the minority were of African or Asian descent, approximating the local 1991 census population.

\section{Medication Protocol}

All children participated in an initial 1-day open trial with a $0.3-\mathrm{mg} / \mathrm{kg}$ dose of methylphenidate to ascertain tolerance, before proceeding with a double-blind, placebo-controlled trial in which all children received each of three doses of methylphenidate $(0.3 \mathrm{mg} / \mathrm{kg}, 0.6 \mathrm{mg} / \mathrm{kg}, 0.9 \mathrm{mg} / \mathrm{kg}$ ) and placebo (lactose powder). Dose was based on child's body weight (to the nearest $2.5 \mathrm{mg}$ ) to facilitate comparison of results with those of previous studies, but recent studies have challenged the idea that that gross body weight is predictive of response to MPH (e.g., Rapport, DuPaul \& Kelly, 1989; Swanson, Cantwell, Lerner, McBurnett, \& Hanna, 1991). Medication 
was given in a single dose and conditions were alternated daily across 4 consecutive days. Order of medication conditions was counterbalanced and determined by random assignment such that an approximately equal number of children received each dose on a given day in the trial. All medication was prepared by the hospital pharmacy and packaged in opaque gelatin capsules to avoid detection of dose and taste. Children, parents, teachers, and research staff were all blind to the medication conditions. Medication was administered by a nurse and testing was conducted between 70 and 120 min postmedication in order to ensure maximum medication effect (Swanson, Kinsbourne, Roberts, \& Zucker, 1978).

\section{Method for Assessing Cognitive Flexibility}

The Change Paradigm. The present study utilized a modification of a method called the stop signal paradigm that requires subjects to stop their response to a simple primary task (usually a forced-choice reaction time task) when given a signal to do so (e.g., Logan et al., 1984). By contrast, the modification that we used - the change paradigm (Logan \& Burkell, 1986) - models the more common and complex real-life situations that require subjects to do something else after stopping the ongoing action (i.e., reengage in another thought and action). In the change paradigm, the stop signal instructs subjects to change from one task to another. Thus, subjects perform the primary task unless a stop signal occurs, which instructs them to inhibit their planned response to the primary task and make a separate overt response to the signal (i.e., the overt response to the signal constitutes the secondary or change task). The stimuli are identical to those of the stop signal paradigm but the number of tasks differ. In the change paradigm there are three tasks (primary, stop, and change task) which involve three sets of processes (primary-task response execution, response inhibition, and response reengagement in the change task), whereas in the stop signal paradigm there are only two tasks and two sets of processes. Thus the cognitive load is greater in the change paradigm.

The stop signal and change paradigms are both based on a theory of inhibitory control in which the ability to inhibit an ongoing thought and action has been formally modeled as a race between two sets of processes: (1) response execution processes that involve stimulus recognition, response choice, and the planning, initiation, and execution of the response to the primary task; and (2) response inhibition processes that are invoked when a stop signal occurs and involve detection of the stop signal and inhibition of the primary-task response. If the inhibitory processes win, the action is inhibited; if the ongoing response process wins, the action runs on to com- 
pletion. The theory provides methods for estimating the latency of the inhibitory control processes (stop signal reaction time or SSRT), even when the act of control is not directly observable (see Appendix). Moreover, the theory provides a rationale and methods for comparing inhibitory control in different subjects under different conditions (e.g., placebo and different doses of MPH), while controlling for potentially confounding factors, such as stimulant-related changes in the response execution processes associated with the primary task. Thus, stimulant effects on inhibitory processes may be separated from concurrent effects on response execution processes. This is important, because one of the most robust effects of MPH is to reduce the variability of response times (Coons, Klorman, \& Borgstedt, 1987; Peloquin \& Klorman, 1986), which in turn might enhance the observed probability of inhibiting ongoing responses (Logan, 1994; Logan \& Cowan, 1984), despite any concomitant adverse effects of the medication on the efficiency of the inhibitory processes. The theoretical assumptions and predictions of the model have been validated empirically in numerous studies of adults (e.g., De Jong et al., 1990; Logan et al., 1984; Osman et al., 1990) and more recently have also been validated in children (Schachar \& Logan, 1990; Schachar et al., in press).

Apparatus and Stimuli. The primary-task stimuli were two uppercase letters presented via an Apple IIe computer that was connected to a specialized Cognitive Testing Station (CTS; Digitry Company Inc., 1984). The CTS software afforded direct and precise control of the stimulus presentation as well as the collection of response times with millisecond accuracy. Each letter, presented one at a time in the center of the screen, was $4 \mathrm{~mm}$ wide and $6 \mathrm{~mm}$ high. When viewed by the subject who sat facing the screen at a distance of $1 \mathrm{~m}$, the angle subtended by each letter was $0.23^{\circ} \times 0.34^{\circ}$.

Each trial began with the presentation of a warning signal. This signal was an asterisk presented at a central fixation point for $500 \mathrm{~ms}$. If was then extinguished and followed immediately by the letter for that trial, which was displayed for $1000 \mathrm{~ms}$. After the letter was extinguished, the screen remained blank for an intertrial interval of $1500 \mathrm{~ms}$. Thus each trial included a period of $2500 \mathrm{~ms}$ in which the subject could respond to either the primary or secondary task in accordance with task demands.

The stop signal was an auditory tone $(1000 \mathrm{~Hz}, 100 \mathrm{~ms}$ in duration) that was also generated by the computer and presented randomly on $25 \%$ of the primary-task trials, balanced across letter type. This signal was presented after the primary-task stimulus but at one of six different intervals $(500,400,300,200,100,0 \mathrm{~ms})$ before the subject's expected primary-task response. The details of the stop signal intervals and the manner in which they were determined are presented in the Appendix. 
Subjects responded to the primary task (choice response) by pressing one of the two left-most buttons on a response box with two fingers on the left hand, as a function of the letter. For the secondary change task (simple response), subjects made an overt response to the tone by pressing a third button on the right of the response box with a finger on the right hand.

Procedure. The primary task involved identifying a single letter from a stimulus set of two letters. A different stimulus set was used at each session: mapping of letters onto the keys was randomized among subjects. Subjects participated in a baseline session and four test sessions on consecutive days. ${ }^{5}$. They performed 11 blocks of 48 trials (total of 528 trials) in each session. The first block consisted of practice trials for the primary task alone; the second block served as practice for the response inhibition and response reengagement task. The remaining nine blocks constituted the test trials and were arranged in sets of three. A short break was scheduled after each set. The two letter stimuli and each stop signal interval occurred equally often in each block and the combination of letter and interval was counterbalanced within each block. A stop signal was presented on $25 \%$ of the trials ( 108 trials), at one of the six equiprobable intervals. Thus, a total of 18 stop signals occurred at each interval. The sequence of primary task stimuli, stop signals, and signal intervals was randomized for each child at every test session. The task lasted approximately 45 min (including instructions and breaks).

Instructions for the primary choice reaction task were given first. Children were told to respond as quickly as possible while maintaining a high level of accuracy. Following the block of practice trials, the children were introduced to the stop signal and change-task response. They were told to try to stop their response to the primary task stimulus whenever they heard the stop signal and immediately respond to the change-task stimulus (i.e., the stop signal). They were also instructed not to delay their responses in anticipation of a stop signal but to make a concerted effort to stop themselves from responding if they heard a stop signal. It was explained that the signals were presented in a way that it would not always be possible to withhold their response. Finally, children were told that, irrespective of their ability to withhold their primary-task response when given a stop signal, they should always respond to that stop signal by pressing the third button on the response box.

Dependent Measures. The change paradigm yields the same measures of the inhibitory processes as the stop signal paradigm and are derived from

${ }^{5}$ Children were first introduced to the change paradigm in an unmedicated state in the baseline session and were given practice during the next session (i.e., the open trial with medication). Because children did not necessarily complete the entire paradigm during these sessions, the baseline and next session did not contribute to the analyzed data. 
the horse-race model for performance in that paradigm: (a) the mean stop signal reaction time (SSRT), which is a derived measure of the latency of the internal response to the stop signal; (b) the slope of the inhibition function [(P)-inhibition slope] that is generated by plotting the observed probability of inhibition as a function of stop signal interval; and (c) ZRFT slope, which reflects the slope of the observed probability of inhibition plotted against a transformation applied to the stop signal interval that corrects for stimulant-related changes in the variability of primary-task response times. This transformation is critical because it provides a means of separating stimulant effects on the inhibitory processes from its effects on the primarytask response processes. These measures are described in more detail in the Appendix and in Logan (1994). If inhibition functions are equivalent when plotted as a function of ZRFT, then differences in the probability of inhibition are due to differences in primary-task variability, as well as any observed differences in SSRT. Differences among the transformed functions (ZRFT slopes) may be attributed to the effects of stimulants on the probability of triggering the inhibitory process or the variability of its latency (or both), as well as any observed differences in the latency of the inhibitory process (SSRT). Thus, beneficial effects of MPH would be indicated by faster SSRT and steeper ZRFT slopes, whereas adverse effects would be indicated by slower SSRT and flatter ZRFT slopes (relative to placebo).

Measures of response reengagement can be derived directly from the speed and variability of the secondary task response (i.e., change task) because the inhibition process does not interfere with response to the change task (Logan \& Burkell, 1986). Response reengagement variables were the latency and variability of the response to the tone, given successful inhibition of the primary-task response (Change-MRT and Change-SD, respectively where $M R T=$ mean response time and $S D=$ standard deviation of response time). Change-task response times and variability were also measured when subjects failed to inhibit their primary-task response and therefore performed two tasks in rapid succession (Dual-MRT and Dual-SD). ${ }^{6}$

Processes involved in the execution of the primary-task response were also measured directly and included: (a) the latency and variability of the response to the primary task (Primary-MRT; Primary-SD); and (b) the accuracy of the response, which was indexed by the percentage of commission errors (\% Commissions: pressing for one letter when the other was presented), the percentage of no response (\% No-response), and percentage

\footnotetext{
${ }^{6}$ In this situation, subjects face a dual task in that responses to two stimuli must be made in rapid succession. According to theory, Dual-MRT will be slower than Secondary-MRT because the primary-task and secondary-task responses compete with each other for access to the motor system, producing interference (Logan \& Burkell, 1986).
} 
of tone-button responses (\% Tone-error: pressing the button for the tone instead of one of the letter buttons).

\section{Methods for Assessing Overt Behavior and Cardiovascular Function}

Children were systematically observed through a one-way mirror while they were completing the change task. Observations were conducted during three 4-min blocks for a total of $12 \mathrm{~min}$. The three blocks were timed to correspond with the three sets of blocked trials in the change paradigm, described previously. Each block consisted of 16 consecutive intervals of 15 sec each, yielding a total of 48 intervals at each test session. During each of the 15-sec intervals, the child was observed continuously for $10 \mathrm{sec}$, and the next $5 \mathrm{sec}$ were used to code and record the behavior. The child's behavior was classified along three dimensions: visual attention to task (on-task vs. off-task), movement (still vs. restless), and vocalization (quiet vs. noisy). Substantial agreement was obtained between two observers for coding offtask $(k=.81)$, restless $(k=.83)$, and noisy $(k=.86)$ across 200 intervals.

A measure of cardiovascular function (heart rate) was included in order to confirm the expected effects of stimulants on this system (e.g., Kelly, Rapport, \& DuPaul, 1988) and to afford an assessment of dose effects across all of the domains (i.e., cognitive, behavioral, cardiovascular) that were included in the seminal study by Sprague and Sleator (1977). Radial pulse, taken for $1 \mathrm{~min}$ with the child seated, was measured three times during each session: immediately prior to medication (Time 0 ) and again at 1 hour (Time 1) and 2 hours (Time 2) following administration of the oral dose. From Time 0 to Time 1, children were seated at a table, coloring or playing quietly with puzzles or board games: children completed the cognitive task from Time 1 to Time 2 .

\section{Statistical Analysis}

The study was designed to test three specific predictions regarding dose effects of stimulants on the cognitive processes of response inhibition and response reengagement (these are summarized in Table II). Accordingly, three focused $F$ tests (contrasts or planned comparisons with numerator $d f=1$ ) were applied to each dependent measure, rather than an overall analysis of variance and an omnibus $F$ test with numerator degrees of freedom greater than 1 which would then need to be followed by multiple post hoc comparisons (e.g., six would be required in the present study). The set of three contrasts was computed based on the coefficients of orthogonal polynomials (trends) so that each contrast was orthogonal to (uncorrelated 
Table II. Alternative Hypotheses and Study Findings for Dose Effects of Methylphenidate on Cognitive Flexibility and Overt Behavior

\begin{tabular}{|c|c|c|c|}
\hline \multirow[b]{2}{*}{ Hypotheses and Study Findings } & \multicolumn{3}{|c|}{ Planned contrasts ${ }^{a}$} \\
\hline & $\begin{array}{l}\text { P vs. } L, M, H \\
\text { (1) }\end{array}$ & $\begin{array}{l}\text { L vs. } \mathrm{H} \\
(2)\end{array}$ & $\begin{array}{l}\text { M vs. } \mathrm{L}, \mathrm{H} \\
(3)\end{array}$ \\
\hline $\begin{array}{l}\text { Hypotheses } \\
\text { 1. Beneficial effects: } \\
\text { Not dose dependent }\end{array}$ & + & - & - \\
\hline $\begin{array}{l}\text { 2. Beneficial effects: } \\
\text { (a) Linear dose curve } \\
\text { (b) Quadratic, U-shaped dose curve }\end{array}$ & $\begin{array}{l}+ \\
+\end{array}$ & $+(\mathrm{H}>\mathrm{L})$ & $\bar{t}$ \\
\hline $\begin{array}{l}\text { 3. Adverse effects: } \\
\text { At high dose }\end{array}$ & + & $+(\mathrm{L}>\mathrm{H})$ & + \\
\hline $\begin{array}{l}\text { Study findings } \\
\text { Cognitive flexibility: }\end{array}$ & & & \\
\hline Response inhibition (SSRT) ${ }^{c}$ & + & - & + \\
\hline Response reengagement (Change-RT) ${ }^{c}$ & + & - & - \\
\hline $\begin{array}{l}\text { Overt behavior: } \\
\text { Motor activity (\% stillness) }\end{array}$ & + & $+(\mathrm{H}>\mathrm{L})$ & - \\
\hline
\end{tabular}

${ }^{a} \mathbf{P}=$ placebo; $\mathrm{L}=$ low dose $(0.3 \mathrm{mg} / \mathrm{kg}) ; \mathrm{M}=$ medium dose $(0.6 \mathrm{mg} / \mathrm{kg}) ; \mathrm{H}=$ high dose $(0.9 \mathrm{mg} / \mathrm{kg})$.

${ }^{b}$ This hypothesis also predicts performance at high dose to be worse than at placebo.

'SSRT = stop signal reaction time; RT = response time.

with) every other contrast. Each contrast was tested against the same error term against which the main effect for medication would be tested in an omnibus $F$ test. Contrast analysis affords much greater statistical power and clearer substantive interpretation of the research results than nonfocused, omnibus tests (for a discussion of orthogonal planned contrasts, see Rosenthal \& Rosnow, 1985).

The shape of the dose-response curve was defined by the pattern of findings across the three contrasts. The first contrast tested for overall effects of medication, whereas the second and third contrasts tested for a linear dose effect and for departures from linear dose effects respectively. ${ }^{7}$ The predicted pattern of findings for each hypothesis is summarized in Table II along with the study findings.

Stimulant effects on cardiovascular function prior to medication and at 1 hour and 2 hours after medication were analyzed with a two-way analysis of variance (ANOVA) with repeated measures across dose (four levels)

${ }^{7}$ The second contrast (low vs. high dose) was really $-1,0,+1$, which was the contrast to test for linearity with three means; and the third contrast was $-1,2,-1$, which was the contrast to test for either departures from linearity or for quadratic trends. 
and time (three levels). Post hoc simple effects ANOVAs and NewmanKeuls tests (Winer, 1971) were conducted to locate differences.

\section{RESULTS}

The mean values for measures of the inhibitory processes, response execution processes associated with the secondary task (i.e., response reengagement), and the primary task that were obtained for each of the three active treatment conditions and placebo are presented in Table III. Also, mean values for measures of overt behavior and cardiovascular function are presented in the lower sections of this table. The observed probability of inhibition at each stop signal interval for each treatment condition is presented in the Appendix (Table A1).

\section{Inhibitory Processes}

The overall effect of the stimulant medication was to accelerate the inhibitory processes (contrast 1 for SSRT: $t(81)=2.349, p<.05$ ), but the dose effects were nonlinear. That is, contrast 3 was significant for SSRT $[t(81)=2.045, p<.05]$, indicating that the greatest improvements in the speed of these central inhibitory processes were obtained at the medium dose, with a relative slowing of the processes at high dose. At medium dose, the inhibitory processes were approximately $40 \mathrm{~ms}$ faster than at either low or high dose and $70 \mathrm{~ms}$ faster than at placebo. However, since contrast 2 was not significant, (i.e., SSRT at low and high dose did not differ), the slowing of SSRT at high dose was relative to medium rather than low dose. As shown in Table II, this pattern of findings for dose effects of MPH on the latency of the inhibitory processes was consistent with the hypothesized pattern for a nonlinear and U-shaped dose-response curve.

The effects of MPH on the inhibition functions were less clear. For example, the $(\mathrm{P})$-inhibition slope increased with increasing dose, but the biggest difference was between placebo and the low dose. The three doses did not differ much from each other. As Table II shows, only contrast 1 was significant $[t(81)=6.618, p<.001]$ for the $(\mathrm{P})$-inhibition slope. Since changes in $(\mathrm{P})$-inhibition may reflect changes in the primary-task response execution processes as well as the inhibitory processes, the results of the analyses for ZRFT slope are crucial to the interpretation. This is because the ZRFT transformation removes effects due to variability in the response execution processes (as well as effects due to differences in SSRT). The analyses of the ZRFT slope showed a similar pattern to the (P)-inhibition 


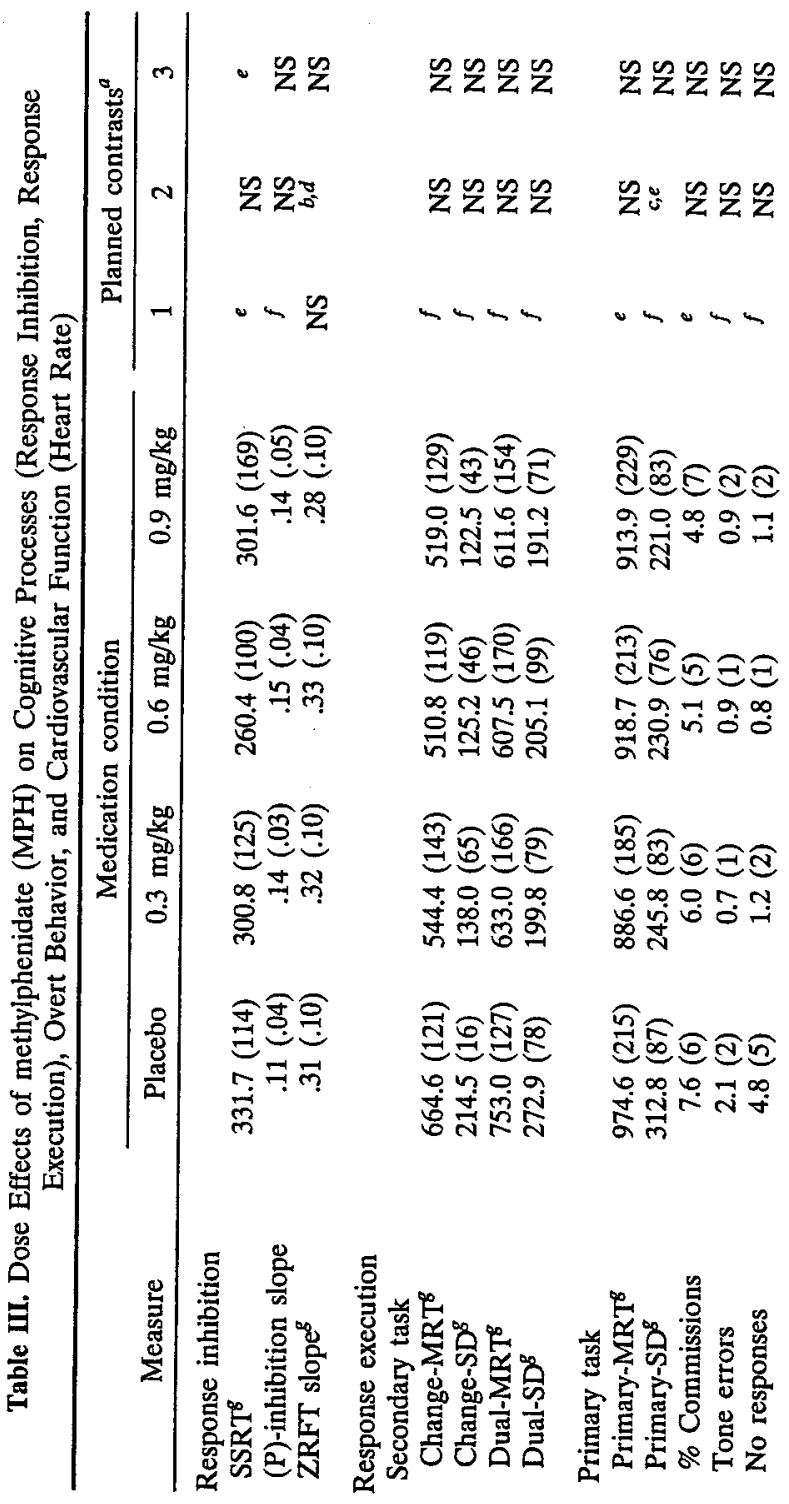




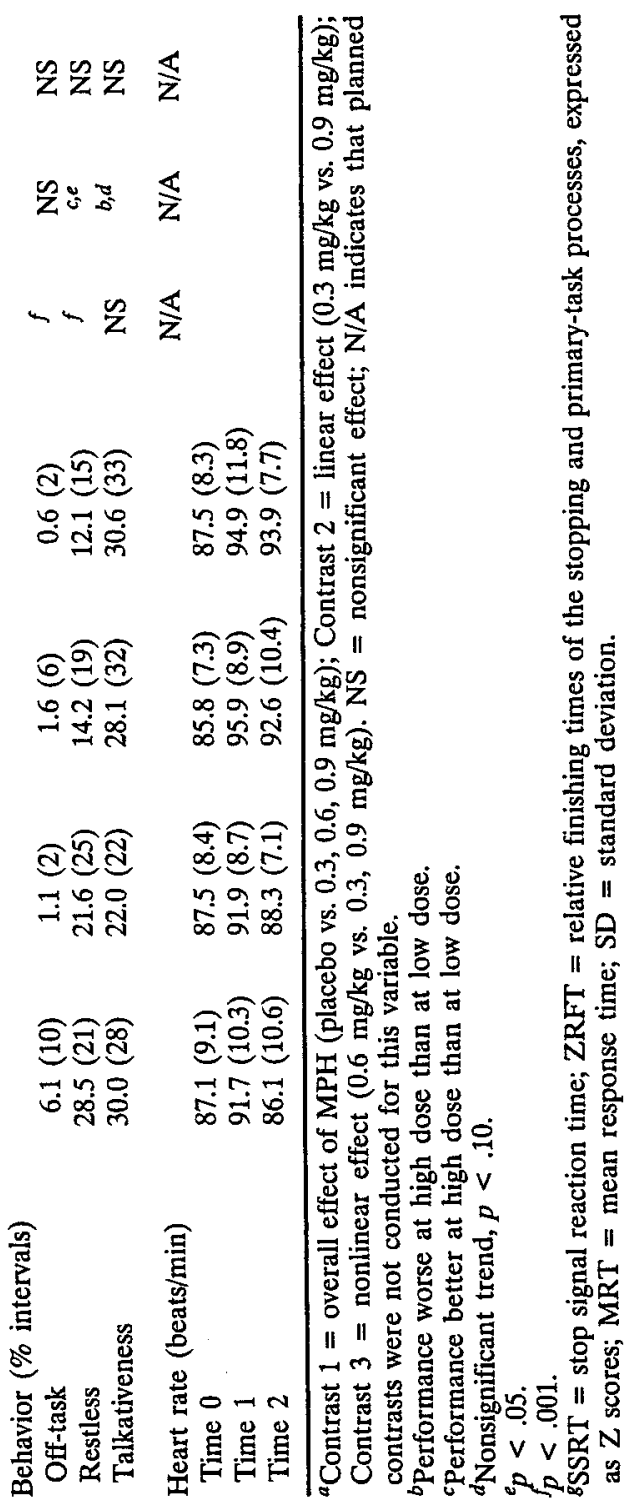


slope, although somewhat attenuated. Table III shows that the ZRFT slope at the high dose was flatter than those at the medium and low doses and at placebo, suggesting that the inhibitory process may be triggered less often at the high dose. However, none of the contrasts were significant [contrast 1: $t(81)=.076, p=.939$; contrast $2: t(81)=1.796, p=.076$; contrast 3: $t(81)=1.123, p=.265$; also the additional contrast between high dose and placebo: $t(27)=1.40, p=.172$ ]. These collective findings suggest that the stimulant-related changes in (P)-inhibition were attributable primarily to the beneficial effects of MPH on the variability of the primary-task response execution processes and on the speed of the inhibitory processes that was indexed by SSRT (see Logan, 1994, for more detailed discussion of ZRFT).

Since there is evidence that MPH effects are highly idiosyncratic and that a sole focus on group data may mask theoretically and clinically important individual response patterns (Douglas et al., 1988; Rapport et al., 1988), medication effects were also examined at the individual level. Approximately $70 \%(n=20)$ of the sample exhibited a dose-related decline at high dose relative to low dose (but not relative to placebo) in terms of their ZRFT slope and 60\% $(n=17)$ exhibited the dose-related decline in SSRT at high dose relative to medium dose. Adverse effects (i.e., indicated by worse performance under at least one of the active medication conditions compared to placebo) were evident in only $14 \%(n=4)$ of the sample for both ZRFT slope and SSRT.

\section{Response Execution Processes}

Our primary interests within the context of the present investigation were dose effects of MPH on response reengagement (i.e., the response execution processes associated with the secondary-change task). As evident from the data presented in Table III, MPH exerted beneficial effects on all of the measures: Secondary-task responses were faster and less variable under active medication conditions compared to placebo. The preceding observations were confirmed by the planned comparisons which revealed that the overall effects of medication (contrast 1 ) were significant for the speed and variability of responses to the secondary task [Change-MRT: $t(81)=8.278, p<.001$; Change-SD: $t(81)=8.749, p<.001$; Dual-MRT: $t(81)=5.996, p<.001$; Dual-SD: $t(81)=4.414, p<.000] .{ }^{8}$ These findings indicate that MPH enhanced the speed with which the response to the

\footnotetext{
${ }^{8}$ Note that Change-MRT was consistently faster than Dual-RT, irrespective of dose $[F(1,27)$ $\left.=21.53, p^{\prime}<.0001\right]$. This finding replicates findings with adults reported by Logan and Burkell (1986) and extends them to child psychopathology.
} 
secondary task was executed, irrespective of whether or not the response to the primary task was inhibited. Since neither contrast 2 nor contrast 3 was significant for these variables, we may conclude that the beneficial effects were not dose-dependent (see Tables II and III).

MPH also exerted beneficial effects on response execution processes associated with the primary task (see Table III). The overall effects of medication (contrast 1) were significant for both the speed and variability of responses to the primary task [Primary-MRT: $t(81)=2.873, p<.01$; Primary-SD $(t(81)=7.782, p<.001]$. Moreover, contrast 2 was also significant for primary-task variability [Primary-SD; $t(81)=1.961, p<.053$ ], indicating that improvements were greater at high dose than at low dose. The pattern of findings for the variability of primary-task response execution processes (Primary-SD) indicated a linear dose-response curve. In contrast to the secondary simple reaction-time task, the primary task had the requirement of choice between two possible responses: Subjects could make three types of primary-task errors (commissions, one errors, and omissions). The overall effect of MPH was to significantly decrease all three types of errors: contrast 1 for percentage of commission errors $[t(81)=2.546, p<$ $.05]$, tone errors $[t(81)=5.521, p<.001]$, and omission errors $[t(81)=$ $6.384, p<.001]$. Neither of the other two planned comparisons was significant for any of these error types, indicating that there was no difference among the three active dose conditions.

\section{Overt Behavior}

As expected, MPH improved overt behavior, particularly in terms of being more visually attentive to the task and less restless during task performance (see Table III). The planned comparisons indicated that the overall effects of medication (contrast 1 ) were beneficial in terms of reducing off-task behavior $[t(81)=4.115, p<.001]$ and restlessness $[t(81)=7.293$, $p<.001]$. Moreover, there was no evidence of nonlinear dose effects. Rather, the additional significant finding for contrast 2 indicated a linear dose-response relationship for motor activity: High dose significantly reduced restlessness compared to low dose $[t(81)=2.497, p<.05]$. By contrast, MPH had no significant influence on talkativeness.

\section{Cardiovascular Function}

Mean values for heart rate (HR) immediately prior to (Time 0 ), and at 1 hour (Time 1) and 2 hours (Time 2) after, ingesting the capsules containing placebo, or low, medium, or high doses of MPH are presented in 
the lower section of Table III. The two-way ANOVA with repeated measures across dose (four levels) and time (three levels) that was conducted on the data revealed significant main effects for dose $[F(3,81)=3.94, p<.05]$, time $[F(2,54)=24.64, p<.001]$ and a significant Dose $\times$ Time interaction $[F(6,162)=4.16, p<.001]$. Post hoc analyses confirmed that there were no differences in HR across the four test sessions prior to medication (i.e., at Time 0), but that at Time 1 changes in HR were linearly related to increases in dose: $\mathrm{HR}$ was significantly faster at the $0.6-\mathrm{mg} / \mathrm{kg}$ dose compared to the $0.3-\mathrm{mg} / \mathrm{kg}$ dose and placebo $(p<.05)$ and also was significantly faster at the $0.9-\mathrm{mg} / \mathrm{kg}$ dose compared to $0.3-\mathrm{mg} / \mathrm{kg}(p<.05)$. Furthermore, these dose-dependent increases in HR persisted. That is, the elevation in HR produced by both the $0.6-$ and $0.9-\mathrm{mg} / \mathrm{kg}$ doses relative to the $0.3-\mathrm{mg} / \mathrm{kg}$ dose and placebo was still evident two hours after ingestion (Time 2: $p<.05$ ).

\section{Comparison of Dose Effects Across Domains}

Trend analyses were conducted to determine the shape of the functions relating dose (placebo, or low, medium, or high doses of $\mathrm{MPH}$ ) and the two critical cognitive processes (inhibitory processes, response reengagement processes), overt behavior, and cardiovascular function. As seen in Table IV, measures of response reengagement (Change-MRT) and behavior (\% stillness) yielded significant linear and quadrative trends. By contrast, only the

Table IV. Trend Analysis of the Relations Between Dose (Placebo, Low, Medium, High Doses of Methylphenidate) and Critical Measures of Cognitive, Behavioral, and Cardiovascular Function

\begin{tabular}{|c|c|c|c|}
\hline \multirow[b]{2}{*}{ Variable $^{b}$} & \multicolumn{3}{|c|}{ Trend $^{a}$} \\
\hline & Linear & Quadratic & Cubic \\
\hline SSRT & 3.23 & $4.91^{c}$ & 1.56 \\
\hline Change-MRT & $53.59^{e}$ & $18.67^{d}$ & 0.19 \\
\hline Behavior (\% stillness) & $52.24^{e}$ & $7.66^{d}$ & 0.14 \\
\hline Heart rate & $36.10^{e}$ & 0.20 & 1.20 \\
\hline $\begin{array}{l}{ }^{a} \text { Degrees of freedom } \\
\text { variable. } \\
{ }^{b} \mathrm{SSRT}=\text { stop signal } \mathrm{r} \\
\text { Change-MRT }=\text { speer } \\
\text { response time) } \\
c_{p}<.05 \\
{ }_{p}<.01 . \\
{ }_{p}<.001 .\end{array}$ & $\begin{array}{l}\text { test ar } \\
\text { time (sp } \\
\text { ponse re }\end{array}$ & $\begin{array}{l}\text { lo to }(1,8 \\
\text { inhibitory } \\
\text { ment (M) }\end{array}$ & $\begin{array}{l}\text { or each } \\
\text { cesses); } \\
=\text { mean }\end{array}$ \\
\hline
\end{tabular}


quadratic trend was significant for inhibitory processes (SSRT), and only the linear trend was significant for cardiovascular function (heart rate).

To facilitate comparison of the shape of the dose-response functions for the four dependent measures across the different domains of function, dose-response data were plotted using standard $T$ scores (with a mean of 50 and $S D$ of 10$).{ }^{9}$ This transformation allowed the variables to be displayed on the same scale. The $T$ scores were calculated so that, for measures of cognitive function and behavior, higher $T$ scores indicated better performance: For cardiovascular function, higher $T$ scores indicated faster heart rate.

The dissociation of dose-related effects across different measures or domains of functioning is illustrated in Fig. 1 in which the mean $T$ scores for each of the selected variables are plotted by dose. In particular, the quadratic and inverted U-shaped function for the central inhibitory proc-

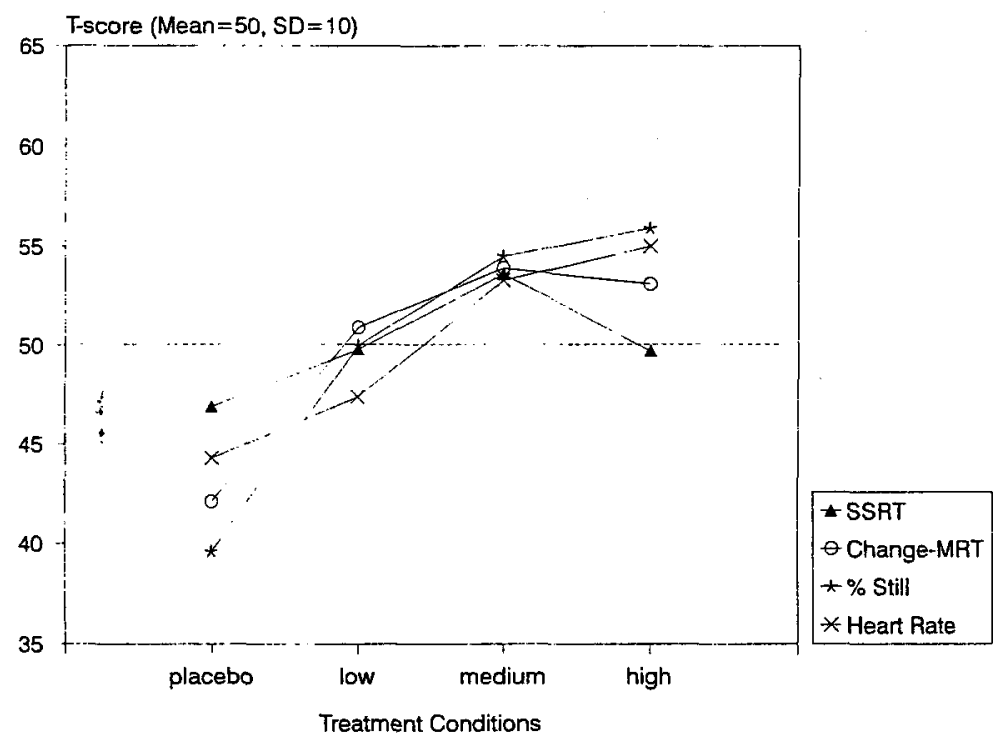

Fig. 1. Dose-response curves of measures of cognitive function (SSRT, Change-MRT), overt behavior (\% stillness), and cardiovascular function (heart rate) for the group of 28 children with ADHD. Means for the group data are expressed as standard $(T)$ scores, so that all measures have an overall mean of 50 and $S D$ of 10. SSRT = stop signal reaction time; $M R T=$ mean response time; $A D H D=$ attention deficithyperactivity disorder.

${ }^{9} T$ scores for each dependent measure were derived based on the performance of the entire sample $(N=28)$, aggregated across placebo and all three active medication conditions to compute an overall mean and standard deviation. 
esses (SSRT) contrasts sharply with the linear dose-response functions for motor activity (\% stillness) and cardiovascular function (heart rate). Note that the function for SSRT is not perfectly quadratic, because at high dose the function has not returned to the initial placebo level. The mean changes in $T$ scores are useful because they illustrated the differential magnitude of effects of stimulants on the two cognitive processes and behavior. For example, MPH produced a maximum change in inhibitory processes of approximately 0.5 of a $S D$ difference, $1.0 S D$ difference for response reengagement processes, and 1.5 SD difference for motor activity.

\section{DISCUSSION}

Our primary interests in the current study were the effects of stimulants on two theoretically independent sets of cognitive processes (i.e., response inhibition, response reengagement) that are implicated in cognitive flexibility. Also, we sought to determine the relative shape of the dose-response functions for overt behavior and these two sets of cognitive processes. The putative effects under investigation were the impairment of cognitive flexibility and a dissociation between dose effects on behavior and cognitive function. We hypothesized that a high dose $(0.9 \mathrm{mg} / \mathrm{kg})$ of MPH would optimize behavioral improvements but impair one or both sets of cognitive processes.

\section{Stimulant Effects on Cognitive Flexibility}

The results of this study do not support the hypothesis that stimulants have an adverse effect on cognitive flexibility: None of the doses of MPH used in this study $(0.3,0.6,0.9 \mathrm{mg} / \mathrm{kg})$ produced an impairment in either response inhibition or response reengagement, relative to placebo. On the contrary, the overall effect of MPH was to accelerate both processes, so that the children were both faster to stop their ongoing actions and faster to immediately execute alternative actions.

An important additional finding was that the latency of the central inhibitory processes was optimized at medium rather than high dose. Moreover, the high dose was no more effective than the low dose in speeding the inhibitory processes and produced a significant slowing of these central control processes compared to the medium dose. Since, there was no evidence that high dose slowed the response execution processes, the findings suggest that the slowing effects of high dose were specific to inhibitory proc- 
esses and were not simply indicative of an overall effect that influenced both response execution and response inhibition processes.

At first glance, the present results for inhibitory processes appear inconsistent with those from our previous study in which we found that improvements in response inhibition were greater at a dose of $1.0 \mathrm{mg} / \mathrm{kg}$ than at $0.3 \mathrm{mg} / \mathrm{kg}$ (Tannock et al., 1989a). The pertinent results from both studies are reproduced in Table V, to facilitate direct comparison. Several important observations can be made from these data. First, the present study provided a more precise characterization of dose effects through the inclusion of an intermediate dose and the data were derived from a larger, more homogeneous, and nonanxious sample of hyperactive children. Second, the data for SSRT at placebo and the high dose of MPH in both studies are highly comparable. This consistency suggests that the findings for SSRT are reliable and therefore that the inclusion of a medium dose in the 1989 study may also have identified a slowing of SSRT at high dose relative to the medium dose. Second, the magnitude of improvement in the latency of both the inhibitory processes (SSRT) and primary-task processes (Primary-MRT) produced by the high dose relative to placebo is smaller for the more demanding change paradigm than for the simple stop signal paradigm (approximately $30 \mathrm{~ms}$ vs. $60 \mathrm{~ms}$, respectively, for SSRT; and $60 \mathrm{~ms}$ vs. $100 \mathrm{~ms}$ for Primary-MRT). Finally, Primary-MRT is slower across all treatment conditions in the change paradigm compared to the stop signal paradigm, even though the stimuli and response demands of the primary task are identical in both paradigms. We cannot conclude from these observations that increasing the cognitive load reduces the magnitude of stimulant effects on response control processes, because this factor was not investigated in either study. However, this effect, which in the extreme

Table V. Mean Latency of the Inhibitory Processes (SSRT) and Response Execution Processes (Primary-MRT) in ADDH/ADHD Children ${ }^{a}$

\begin{tabular}{|c|c|c|c|c|}
\hline & \multicolumn{4}{|c|}{ Treatment condition } \\
\hline & Placebo & $0.3 \mathrm{mg} / \mathrm{kg}$ & $0.6 \mathrm{mg} / \mathrm{kg}$ & $0.9 \mathrm{mg} / \mathrm{kg}$ \\
\hline \multicolumn{5}{|l|}{ SSRT (in ms) } \\
\hline 1989 Study $(n=12)^{b}$ & 354 & 351 & N/A & 297 \\
\hline Present study $(n=28)$ & 332 & 301 & 260 & 302 \\
\hline \multicolumn{5}{|l|}{ Primary-MRT (in ms) } \\
\hline 1989 Study $(n=12)$ & 904 & 841 & N/A & 802 \\
\hline Present study $(n=28)$ & 975 & 887 & 919 & 914 \\
\hline
\end{tabular}


might impair the central control processes, remains a possibility and requires further investigation.

\section{Dose-Response Functions}

The present results provide partial support for the hypothesis that dose-response curves for behavior and cognitive function differ. For example, the dose-response function for motor activity (i.e., one index of overt behavior) was primarily linear, indicating that increases in dose (at least up to a dose of $0.9 \mathrm{mg} / \mathrm{kg}$ ) result in greater reductions in motoric restlessness. By contrast, the dose-response function for the central inhibitory processes (response inhibition) was nonlinear and U-shaped. That is, as previously described, the latency of these cognitive control processes (SSRT) was optimally enhanced at the medium dose: the processes were significantly slower at both the low dose and the high dose, relative to the medium dose.

It is important to note, however, that the concurrent dose effects on different measures within a given domain were not necessarily comparable. For example, unlike measures of the inhibitory processes, indices of response reengagement processes did not exhibit U-shaped dose-response curves. On the contrary, indices of these response execution processes (and those associated with the primary-task response) yielded dose-response functions that formed the shape of a dog-leg (i.e., an inverted $L$ shape). Our findings suggest that dose-response curves vary across different measures both within and across domains and that the polarized view of dissociated dose-response curves for behavior and cognition (e.g., Sprague \& Sleator, 1977) was overly simplistic.

Finally, in light of recent data suggesting a link between response inhibition and inhibition of the cardiac cycle (Jennings, van der Molen, Brock, \& Somsen, 1992), a comment is warranted on the concurrent dose effects on heart rate observed in this study. In contrast to the dose-response function for response inhibition where the disjuncture occurred between the medium and high doses, the separation of dose effects on heart rate occurred between the low dose and the two higher doses. That is, the low dose produced small increases in heart rate that were not significant and dissipated within 2 hours of receiving the medication. By contrast, the medium and high doses both produced modest elevations in heart rate (mean increase of four and five beats/min at medium and high doses, respectively) that were still evident 2 hours later. These dose- and time-dependent changes in heart rate are consistent with previous research (e.g., Kelly et al., 1988; Tannock et al., 1989b) but are modest in comparison to the elevations induced by exercise or other mental stressors, such as a competitive video game or men- 
tal arithmetic tasks (e.g., Aman \& Werry, 1975; Boyce \& Chesterman, 1990). Since there is no evidence that heart rate remains significantly elevated during extended stimulant treatment (Safer, 1992), the increases in heart rate observed in the present study were considered to be clinically unimportant. The possible link between stop signal inhibition and cardiac inhibition is intriguing, but our methods were not designed to investigate the precise link between response control and the cardiac cycle. Further research is necessary to determine the concurrent effects of stimulants on cardiac activity and cortical activity during response inhibition.

\section{Theoretical and Clinical Implications}

The observed dissociation between dose effects on response inhibition and response execution adds to the body of empirical evidence supporting the theoretical assumptions that these two sets of cognitive processes are independent (De Jong et al., 1990; Jennings et al., 1992; Logan et al., 1984). Our findings suggest the two sets of processes exhibit different sensitivities to psychostimulants.

Our findings that a high dose of MPH was less effective than a lower dose in enhancing the central inhibitory processes are consistent with previous findings of apparent decrements in other aspects of cognitive function at high dose relative to lower doses (Douglas et al., 1988; Peeke, Halliday, Callaway, Prael, \& Reus, 1984; Rapport, Stoner, DuPaul, Birmingham, \& Tucker, 1985; Sprague \& Sleator, 1977). On the other hand, our results are at odds with numerous well-controlled studies of dose effects of stimulants on cognitive function that have failed to demonstrate inverted $U$ shaped relationships at the group level of analysis. A review of those studies (Rapport \& Kelly, 1991) indicated that optimum performance on most cognitive tasks was obtained at high dose.

What could account for the apparent ineffectiveness of high dose relative to lower doses? First, this pattern of findings may indicate that inhibitory processes would be at risk for adverse effects from stimulant medication if either the dose or level of cognitive demand, or both, were increased. It is noteworthy in this regard that previous evidence of performance decrements at high dose has been found on cognitively complex tasks or on the most difficult levels of a task (e.g., Douglas et al., 1988; Tannock \& Schachar, 1992). Moreover, given that the effect was to slow the inhibitory processes (albeit relative to a lower dose rather than placebo) rather than the execution processes, it is reasonable to speculate that a stimulant-induced decrement in inhibitory control may underlie previous clinical and empirical reports of perseverative phenomenology in hyperac- 
tive children receiving stimulant treatment (e.g., Laufer et al., 1957; Tannock \& Schachar, 1992).

An alternative (but not incompatible) interpretation of the decreased effectiveness of high dose in enhancing response inhibition is that the time course of effects of high doses on these processes may differ from that of lower doses. Thus, the maximum changes in response inhibition (irrespective of whether they were beneficial or adverse) at high dose may have occurred either earlier or later than the period across which we measured dose effects in the present study. Consistent with this hypothesis are the reports of an interaction between dose and time course of effects on other measures of cognitive performance (Swanson et al., 1991; Tannock, Schachar, \& Logan, 1993). Stimulant dose, the time course of stimulant action, task complexity, and the type of cognitive processes involved are likely to be interdependent. Thus, if we are to understand stimulant effects on cognitive flexibility, it will be essential to study these factors simultaneously in future studies.

Evidence of dissociated dose effects on different aspects of behavior and cognitive function are particularly relevant to clinicians in terms of prescribing and monitoring stimulant treatment. In clinical practice, both the prediction of stimulant response and the determination of an optimal dose are typically determined from a single measure (usually from parent or teacher descriptions of changes in the child's behavior). This practice is predicated on the assumption that stimulants have a unitary mechanism of action and that dose-response functions for behavior are assumed to be comparable to those for other domains of functioning, such as cognitive function, learning, and academic achievement. This assumption is challenged by the present findings of different dose-response curves for different measures within and across various domains of function. Moreover, because the emphasis in determining dose levels is on reduction of overt activity and other disruptive behavior, many children may be at risk for receiving a dose that is optimal for reducing motor activity but relatively ineffective in enhancing response inhibition. This possibility is of particular concern, because this central control process is known to be deficient in children with ADHD (e.g., Schachar \& Logan, 1990; Schachar et al., in press). These findings highlight the importance of assessing stimulant response by measuring different aspects of function across the entire period of stimulant action. Clinicians are advised to include measures of stimulant response that provide some index of cognitive function (e.g., Academic Performance Rating Scale: DuPaul, Rapport, \& Periello, 1991).

The findings reported herein must be interpreted with caution because of several factors. First, the findings are based on an acute and single challenge at each dose level. Moreover, the observed decline in response 
inhibition occurred at a single dose ranging from $20 \mathrm{mg}$ to $35 \mathrm{mg}$ (i.e., 0.9 $\mathrm{mg} / \mathrm{kg}$ ), which is likely at the upper end of the range used in current clinical practice (i.e., recommended maximum dose of $60 \mathrm{mg}$ per day in divided doses). It is unknown whether the acute response to MPH at a particular dose predicts a similar degree of change once the child is placed on a maintenance regimen using the same parameters of dose. Moreover, it is important to note that the observed decrement in the inhibitory processes at high dose occurred relative to lower-dose conditions and not to placebo. Finally, statistical evidence of a dose-related decline in one aspect of cognitive function based on a laboratory paradigm does not necessarily imply clinical importance: The change paradigm has no established external validity (e.g., with cognitive demands associated with the classroom).

\section{CONCLUSIONS}

Results from the present investigation do not support the hypothesis regarding the adverse effects of stimulant treatment on cognitive flexibility. They do, however, indicate that the dose-response curve for central inhibitory processes is U-shaped, whereas that for motor activity is primarily linear. It is unknown what accounts for an apparent decrease in effectiveness of high dose in enhancing inhibitory control. We argue that further doseresponse studies on cognitive function are urgently needed and that inhibitory processes provide an important locus for investigation from both a theoretical and clinical perspective.

\section{APPENDIX}

\section{Setting the Stop Signal Interval}

According to the model of Logan and colleagues (Logan \& Cowan, 1984; Logan et al., 1984), the probability that a response will be inhibited given a signal to stop depends on the speed and variability of the go process (i.e., primary-task response) and the speed and variability of the inhibition process, as well as the stop signal interval. If the stop signal is presented early enough, subjects will inhibit all the time; if it is presented late enough, they will respond all the time. The critical issue is to choose and set the stop signals at intervals to capture the part of the inhibition function that is the most informative theoretically - where the probability of inhibiting changes smoothly from 0 to 1 . Several methods are available (see Logan et al., 1984), but in this study stop signal intervals were set in relationship 
to each child's mean primary-task reaction time. The rationale was that the stop signal interval can control for individual differences in primary-task reaction time and its expected influence by stimulants.

More specifically, the intervals were set dynamically by tracking each subject's mean reaction time and presenting stop signals at fixed intervals before this point in time. In the present study, the tone was presented after the primary-task stimulus but either $500,400,300,200,100$, or $0 \mathrm{~ms}$ before the subject's mean response time. (See Table A1.) A subject's mean primary-task response time (Primary-MRT) calculated in the first block of practice trials was used to set stop signals at the six intervals for the second block. Primary-MRT for the nonsignal trials for the second block was then used to set the delays for the third block, and so on. The dynamic tracking of each subject's mean reaction time and concomitant adjustment of stop switch signal intervals means that intervals for a given block depended only upon performance in the immediately preceding block.

\section{(P)-Inhibition Slope}

Inhibition functions relate the probability of inhibiting (P-inhibition) to stop signal intervals. These functions are important theoretically, because they reflect the outcome of the race between the inhibitory processes and the response execution processes. $(\mathrm{P})$-inhibition is calculated by determin-

Table A1. Probability of Inhibition at Each Stop Signal Interval for Placebo and Three Doses of Methylphenidate ${ }^{a}$

\begin{tabular}{lcccc}
\hline & \multicolumn{4}{c}{ Treatment condition } \\
\cline { 2 - 5 } Stop signal interval & Placebo & $0.3 \mathrm{mg} / \mathrm{kg}$ & $0.6 \mathrm{mg} / \mathrm{kg}$ & $0.9 \mathrm{mg} / \mathrm{kg}$ \\
\hline MRT-500 & .67 & .75 & .79 & .75 \\
MRT-400 & .53 & .66 & .73 & .70 \\
MRT-300 & .47 & .56 & .61 & .59 \\
MRT-200 & .33 & .34 & .38 & .37 \\
MRT-100 & .21 & .23 & .23 & .23 \\
MRT-0 & .15 & .10 & .12 & .11 \\
\hline
\end{tabular}

${ }^{a}$ Figures in table are for the mean corrected (P)-inhibition (see Appendix for details of correction).

${ }^{b}$ Mean primary-task response time (MRT) minus the specified interval in milliseconds (e.g., $500 \mathrm{~ms}$ ). 
ing the proportion of stop signal trials at a given interval that the subject successfully inhibited [e.g., if a subject inhibited 12 out of the 18 stop signal trials at the stop signal interval of Primary-MRT minus $500 \mathrm{~ms}$, then (P)inhibition would be .67 for that interval]. In this study, we plot corrected (P)-inhibition by stop signal interval. A correction is necessary because children in general, and children with a diagnosis of ADHD in particular, often fail to respond some of the time either as a result of an active strategy or inattentiveness. Since some of these omissions may occur on stop signal trials, the observed $(\mathrm{P})$-inhibition may reflect both omissions and true response inhibition. This effect extended across all stop signal intervals would increase the height and steepness of inhibition functions. Consequently, (P)-inhibition at each interval was corrected for the percent of omission observed on nonsignal trials, using the following formula:

$$
y=\frac{x-o}{(N-q)-o}
$$

where $y$ is the corrected number of inhibited trials at a specific interval, $x$ is the observed number of inhibited trials at that interval, and $o$ is the correction for the number of omissions. $N$ is the total number of stop signal trials at each interval ( $N=18$ in the present study) and $q$ is a correction for the number of trials at each interval that were executed but in an inappropriate fashion (e.g., "early" responses that occurred within $250 \mathrm{~ms}$ of the primary-task stimulus, response to the tone followed by response to the primary-task stimulus). We calculated the correction for the number of omissions $(o)$ according to the following formula:

$$
o=\frac{n}{P-t}
$$

where $n$ is the number of no responses on nonsignal primary-task trials, $P$ is the total number of primary-task trials ( $P=324$ in this study), and $t$ is the number of responses involving presses of the beep button on nonsignal primary-task trials. We subtracted $t$ from the total number of primary-task trials because it indicated that subjects perceived the primary-task stimulus but incorrectly responded to the secondary task. All subsequent analyses were conducted on the corrected probability of inhibition.

\section{Stop Signal Reaction Time}

The latency of the internal response to the stop signal (SSRT) cannot be observed directly, but it can be estimated. Logan (1994) described three methods: In this study we used Logan's (1981) method that assumes SSRT 
is constant. SSRT is the difference between the point at which the stop signal is presented and the point at which the inhibitory process finished. Presentation of the stop signal can be determined from the experimental protocol (in this case Primary-MRT - $500 \mathrm{~ms}$, MRT - $400 \mathrm{~ms}$, etc.). The point at which the inhibitory process finished can be calculated from the observed distribution of primary-task response times (on trials without stop change signals) and the probability of inhibition at a given stop signal interval (see Logan \& Cowan, 1984). More specifically, the distribution of primary-task response times on nonsignal trials was rank-ordered and the $n$th reaction time was selected, where $n$ was obtained by multiplying the number of responses in the primary-task distribution (324 nonsignal trials in the present study) by 1 minus the corrected probability of inhibiting at a given delay. The $n$th reaction time estimates the time at which the stopping process finished, relative to the onset of the go signal. SSRT was estimated by subtracting the stop signal interval, defined in this study as MRT -the set interval [i.e., $n$th RT-(MRT -500$)$ ] from the $n$th reaction time. This process was repeated for each stop signal interval and results were averaged across stop signal intervals to yield a mean SSRT for each subject under each treatment condition.

\section{ZRFT-Slope}

Changes in the slope of the inhibition functions may not necessarily reflect changes in the inhibitory processes. For example, differences in the slope of the (corrected) inhibition functions could be attributable to stimulant-related effects on various parameters associated with the inhibitory process per se (i.e., SSRT, variability of SSRT, probability of triggering the inhibitory process) or on a parameter of the response execution process variability of primary-task reaction time. Since one of the most robust effects of stimulants is to reduce the variability of response times, it is essential to separate dose effects on the inhibitory process per se from those on the response execution processes associated with the primary-task response. To do this, a transformation is applied to the inhibition function that permits examination of the effect of variability in stop signal reaction time and of probability of triggering a stopping response on the probability of inhibition (see Logan \& Cowan, 1984). Probability of inhibition is plotted as a function of a $Z$ score that represents the relative finishing time of the primary-task and inhibition processes in standard deviation units, using the primary-task reaction times to define the units delay minus SSRT:

$$
\text { ZRFT }=\frac{\text { delay }- \text { SSRT }}{\text { SDRT }}
$$


where ZRFT is the relative finishing times of the stopping and the primary-task processes, expressed as a $Z$ score; delay is the interval between the presentation of the stop signal and the subject's mean primary-task reaction time; SSRT is the estimated stop signal response time; and SDRT is the standard deviation of the primary-task response times.

If the inhibition function produced under the different medication conditions (i.e., placebo and low, medium, or high dose of MPH) can be aligned with the ZRFT transformation, then differences in (P)-inhibition are due to differences in the standard deviation of primary-task reaction time and in SSRT. Any differences in SSRT should be interpreted, because they may reflect specific changes in the inhibitory processes. If the inhibition function cannot be completely aligned with the ZRFT transformations, then we can conclude that the shallower functions represent deficiencies in inhibitory control; either the inhibitory process has more variability, or it is triggered less often.

\section{REFERENCES}

Aman, M. G., \& Werry, J. S. (1975). The effects of methylphenidate and haloperidol on the heart rate and blood pressure of hyperactive children with special reference to time of action. Psychopharmacologia, 43, 163-188.

American Academy of Pediatrics. (1987). Committee on children with disabilities, committee on drugs. Medication for children with an attention deficit disorder. Pediatrics, 80, 758-760.

American Psychiatric Association. (1987). Diagnostic and statistical manual of mental disorders (3rd ed., rev.). Washington, DC: Author.

Boyce, W. T., \& Chesterman, E. (1990). Life events, social support and cardiovascular reactivity in adolescence. Journal of Developmental and Behavioral Pediatrics, 11, 105-111.

Brown, R. T., \& Sleator, E. K. (1979). Methylphenidate in hyperkinetic children: Differences in dose effects on impulsive behavior. Pediatrics, 64, 408-411.

Conners, C. K. (1973). Rating scales for use with drug studies with children. Psychopharmacology Bulletin, 19, 24-29.

Coons, H. W., Klorman, R., \& Borgstedt, A. D. (1987). Effects of methylphenidate on adolescents with a childhood history of attention deficit disorder: II. Information processing. Journal of the American Academy of Child and Adolescent Psychiatry, 26, 368-374.

De Jong, R., Coles, M. G. H., Logan, G. D., \& Gratton, G. (1990). In search of the point of no return: The control of response processes. Journal of Experimental Psychology: Human Perception and Performance, 16, 164-182.

Digitry Company Inc. (1984). Cognitive Testing Station [Apparatus and computer software]. (Available from the company at 108 High Street, Portland, ME 04102.)

Douglas, V. I., Barr, R. G., Amin, K., O’Neill, M. E., \& Britton, B. G. (1988). Dosage effects and individual responsivity to methylphenidate in attention deficit disorder. Journal of Child Psychology and Psychiatry, 29, 453-475.

DuPaul, G. J., \& Barkley, R. A. (1990). Medication therapy. In R. A. Barkley (Ed.), Attention deficit hyperactivity disorder: $A$ Handbook for diagnosis and treatment (pp. 573-612). New York: Guilford Press. 
DuPaul, G. J., Rapport, M. D., \& Periello, L. M. (1991). Teacher ratings of academic skills: The development of the Academic Performance Rating Scale. School Psychology Review, $20,284-300$.

Dyme, I. Z., Sahakian, B. J., Golinko, B. E., \& Rabe, E. (1982). Perseveration induced by methylphenidate in children: Preliminary findings. Progress in Neuro-Psychopharmacology and Biological Psychiatry, 6, 269-273.

Flintoff, M. M., Barron, R. W., Swanson, J. M., Ledlow, A., \& Kinsbourne, M. (1982). Methylphenidate increases selectivity of visual scanning in children referred for hyperactivity. Joumal of Abnormal Child Psychology, 10, 145-161.

Grattan, L. M., \& Eslinger, P. J. (1990). Higher cognition and social behavior: Cognitive flexibility and empathy after brain injury. Neuropsychology, 3, 175-185.

Jacobvitz, D., Sroufe, L. A., Stewart, M., \& Leffert, N. (1990). Treatment of attentional and hyperactivity problems in children with sympathomimetic drugs: A comprehensive review. Journal of the American Academy of Child and Adolescent Psychiatry, 29, 677-688.

Jastak, S., \& Wilkinson, G. S. (1984). Manual for the Wide Range Achievement Test (revised). Wilmington, DE: Jastak.

Jennings, J. R., van der Molen, M. W., Brock, K., \& Somsen, R. J. M. (1992). On the synchrony of stopping motor responses and delaying heartbeats. Journal of Experimental Psychology: Human Perception and Performance, 2, 422-436.

Kelly, K. L., Rapport, M. D., \& DuPaul, G. J. (1988). Attention deficit disorder and methylphenidate: A multi-step analysis of dose-response effects on children's cardiovascular functioning. International Clinical Psychopharmacology, 3, 167-181.

Laufer, M. W., Denhoff, E., \& Riverside, R. I. (1957). Hyperkinetic behavior syndrome in children. Journal of Pediatrics, 50, 463-474.

Lezak, M. D. (1983). Neuropsychological assessment. New York: Oxford University Press.

Logan, G. D. (1981). Attention, automaticity, and the ability to stop a speeded choice response. In J. Long \& A. D. Baddeley (Eds.), Attention and performance IX. Hillsdale, New Jersey: Lawrence Erlbaum.

Logan, G. D. (1994). On the ability to inhibit thought and action: A users' guide to the stop signal paradigm. In D. Dagenbach \& T. H. Carr (Eds.), Inhibitory processes in attention, memory, and language. San Diego: Academic Press.

Logan, G. D., \& Burkell, J. (1986). Dependence and independence in responding to double stimulation: A comparison of stop, change and dual-task paradigms. Journal of Experimental Psychology: Human Perception and Performance, 12, 549-563.

Logan, G. D., \& Cowan, W. B. (1984). On the ability to inhibit thought and action: A theory of an act of control. Psychological Review, 91, 295-327.

Logan, G. D., Cowan, W. B., \& Davis, K. A. (1984). On the ability to inhibit simple and choice reaction time responses: A model and a method. Journal of Experimental Psychology: Human Perception and Performance, 10, 276-291.

Osman, A., Kornblum, S., \& Meyer, D. E. (1990). Does response programming necessitate response execution? Journal of Experimental Psychology: Human Perception and Performance, 10, 358-377.

Peeke, S., Halliday, R., Callaway, E., Prael, R., \& Reus, V. (1984). Effects of two doses of methylphenidate on verbal information processing in hyperactive children. Journal of Clinical Psychopharmacology, 4, 82-88.

Pelham, W. E., Atkins, M., \& Murphy, H. A. (1981, September). ADD with and without hyperactivity: Parent, teacher and peer rating correlates. In W. E. Pelham (Chair), DSM-III category of attention deficit disorder: Rational, operation, and correlates. Symposium conducted at the meeting of the American Psychological Association, Los Angeles.

Pelham, W. E., Bender, M. E., Caddell, J., Booth, S., \& Moorer, S. H. (1985). Methylphenidate and children with attention deficit disorder. Archives of General Psychiatry, 42, 948-952.

Peloquin, L. J., \& Klorman, R. (1986). Effects of methylphenidate on normal children's mood, event related potentials, and performance in memory scanning and vigilance. Journal of Abnormal Psychology, 95, 88-98. 
Peters, J. E., Dykman, R. A., Ackerman, P. T., \& Romine, J. S. (1974). The special neurological examination. In C. K. Conners (Ed.), Clinical use of stimulant drugs in children. New York: Elsevier.

Pliszka, S. R., \& Borcherding, S. H. (1994). The stop signal task in attention deficit hyperactivity disorder children with and without comorbid anxiety. Manuscript submitted for publication.

Rapoport, J. L., Buschbaum, M. S., Zahn, T. P., Weingartner, H., Ludlow, C., \& Mikkelsen, E. J. (1978). Dextroamphetamine: Cognitive and behavioral effects in normal prepubertal boys. Science, 199, 560-563.

Rapport, M. D., DuPaul, G. J., \& Kelly, K. L. (1989). Attention deficit hyperactivity disorder and methylphenidate: The relationship between gross body weight and drug response in children. Psychopharmacology Bulletin, 25, 285-290.

Rapport, M. D., \& Kelly, K. L. (1991). Psychostimulant effects on learning and cognitive function: Findings and implications for children with attention deficit hyperactivity disorder. Clinical Psychology Review, 11, 61-92.

Rapport, M. D., Stoner, G., DuPaul, G. J., Birmingham, B. K., \& Tucker, S. (1985). Methylphenidate in hyperactive children: Differential effects of dose on academic learning, and social behavior. Journal of Abnormal Child Psychology, 13, 227-244.

Rapport, M. D., Stoner, G., DuPaul, G. J., Kelly, D. L., Tucker, S. B., \& Schoeler, T. (1988). Attention deficit disorder and methylphenidate: A multilevel analysis of dose-response effects on children's impulsivity across settings. Journal of the American Academy of Child and Adolescent Psychiatry, 27, 60-69.

Robbins, T. W., \& Sahakian, B. J. (1979). Paradoxical effects of psychomotor stimulant drugs in hyperactive children from the standpoint of behavioral pharmacology. Neuropharmacology, 18, 931-950.

Rosenthal, R., \& Rosnow, R. L. (1985). Contrast analysis: Focused comparisons in the analysis of variance. Cambridge, England: Cambridge University Press.

Rutter, M., Tizard, J., \& Whitmore, K. (Eds.). (1981). Education, health and behavior: Psychological and medical study of childhood development. Huntington, New York: Krieger. (Original work published 1970)

Safer, D. J. (1992). Relative cardiovascular safety of psychostimulants used to teat attentiondeficit hyperactivity disorder. Joumal of Child and Adolescent Psychopharmacology, 2, 279-290.

Safer, D. J., \& Krager, J. M. (1988). A survey of medication treatment for hyperactive/ inattentive students. Joumal of the American Medical Association, 260, 2256-2258.

Schachar, R. J., \& Logan, G. D. (1990). Impulsivity and inhibitory control in normal development and childhood psychopathology. Developmental Psychology, 26, 710-720.

Schachar, R., Rutter, M., \& Smith, A. (1981). The characteristics of situationally and pervasively hyperactive children: Implications for syndrome definition. Journal of Child Psychology and Psychiatry, 22, 375-392.

Schachar, R., \& Tannock, R. (1990). Teacher Telephone Interview. Unpublished manuscript, The Hospital for Sick Children, Department of Psychiatry, Toronto.

Schachar, R., \& Tannock, R. (1993). Childhood hyperactivity and psychostimulants: A review of extended treatment studies. Journal of Child and Adolescent Psychopharmacology, 3, 81-97.

Schachar, R. J., Tannock, R., \& Logan, G. (1993). Inhibitory control, impulsiveness and attention deficit hyperactivity disorder. Clinical Psychology Review, 13, 721-739.

Schachar, R., Tannock, R., Marriott, M., \& Logan, G. (in press). Deficient inhibitory control in attention deficit hyperactivity disorder. Journal of Abnormal Child Psychology.

Schachar, R. J., \& Wachsmuth, R. (1989). Parent Interview for Child Symptoms - Revised $D S M-I I I-R$. Unpublished manuscript, Hospital for Sick Children, Department of Psychiatry. Toronto.

Solanto, M. V. (1984). Neuropharmacological basis of stimulant drug action in attention deficit disorder with hyperactivity: A review and synthesis. Psychological Bulletin, 95, 387-409.

Solanto, M., \& Conners, C. (1982). A dose-response and time-action analysis of autonomic and behavioral effects of methylphenidate in attention deficit disorder with hyperactivity. Psychophysiology, 19, 658-667. 
Solanto, M. V., \& Wender, E. H. (1989). Does methylphenidate constrict cognitive functioning? Journal of the American Academy of Child and Adolescent Psychiatry, 28, 897-902.

Sprague, R. L., \& Gadow, K. D. (1976). The role of the teacher in drug treatment. School Review, 85, 109-140.

Sprague, R. L., \& Sleator, E. K. (1977). Methylphenidate in hyperkinetic children: Differences in dose effects on learning and social behavior. Science, 198, 1274-1276.

Sroufe, L. A., \& Stewart, M. A. (1973). Treating problem children with stimulant drugs. New England Journal of Medicine, 289, 407-413.

Swanson, J. M., Cantwell, D., Lerner, M., McBurnett, K., \& Hanna, G. (1991). Effects of stimulant medication on learning in children with ADHD. Journal of Leaming Disabilities, $24,219-230$.

Swanson, J. M., \& Kinsbourne, M. (1979). The cognitive effects of stimulant drugs on hyperactive children. In G. A. Hale \& M. Lewis (Eds.), Attention and cognitive development (pp. 249-274). New York: Plenum Press.

Swanson, J. M., Kinsbourne, M., Roberts, W., \& Zucker, K. (1978). Time-response analysis of the effects of stimulant medication on the learning ability of children referred for hyperactivity. Pediatrics, 61, 21-29.

Tannock, R., \& Schachar, R. (1992). Methylphenidate and cognitive perseveration in hyperactive children. Journal of Child Psychology and Psychiatry, 33, 1217-1228.

Tannock, R., Schachar, R. J., Carr, R. P., Chajczyk, D., \& Logan, G. D. (1989a). Effects of methylphenidate on inhibitory control in hyperactive children. Journal of Abnormal Child Psychology, 17, 473-491.

Tannock, R., Schachar, R. J., Carr, R. P., \& Logan, G. D. (1989b). Dose-response effects of methylphenidate on academic performance and overt behavior in hyperactive children. Pediatrics, 84, 648-657.

Tannock, R., Schachar, R. J., \& Logan, D. G. (1993). Does methylphenidate induce overfocusing in hyperactive children? Joumal of Clinical Child Psychology, 22, 28-41.

Walsh, K. W. (1978). Neuropsychology, a clinical approach. New York: Livingstone.

Wechsler, D. (1974). Manual for the Wechsler Intelligence Scale for Children-Revised. San Antonio: The Psychological Corporation.

Winer, B. J. (1971). Statistical principles in experimental design. New York: McGraw-Hill. 